\title{
COMPORTAMIENTO DE LAS ENFERMEDADES TRANSMITIDAS POR ALIMENTOS Y ESTRATEGIAS DE MEJORAMIENTO EN CUATRO MUNICIPIOS DEL QUINDIO, COLOMBIA.
}

\author{
BEHAVIOR OF FOODBORNE DISEASES AND IMPROVEMENT STRATEGIES IN \\ FOUR MUNICIPALITIES OF THE QUINDIO, COLOMBIA.
}

María Cristina Montenegro Benavides ${ }^{1}$, Paula Alejandra Osorio²

1. Ingeniera de Alimentos. Fundación Universitaria del Área Andina Seccional Pereira. grupo ZIPATEFI

2. Salubrista Ocupacional. Fundación Universitaria del Área Andina Seccional Pereira. grupo ZIPATEFI

Correspondencia del Autor: Cra. 39 \#19-138 Barrio Palermo Pasto Nariño.

E-mail: macrismo99@hotmail.com.

\begin{abstract}
RESUMEN
Durante dos años se obtuvo información de la condición epidemiológica de las enfermedades transmitidas por alimentos en cuatro municipios del Quindío, Colombia. Durante este tiempo, se presentaron 32 casos colectivos que involucraron 240 personas de diferentes edades de Calarcá, Tebaida, Montenegro y Quimbaya lo cual permitió proponer estrategias para promover la investigación de brotes como la implementación de una estrategia de vigilancia epidemiológica; las variables estudiadas se determinaron de acuerdo a la ficha de notificación de casos colectivos: género, edad, municipio, grupo poblacional, fecha de exposición, hospitalización, alimento consumido, signos y síntomas y resultados de laboratorio. En dicho estudio se analizó la información registrada en la ficha de notificación y se encontró que el registro se hace de manera incompleta, lo que demuestra fallas en los procesos de información continua de brotes de ETA. No fue posible realizar investigación de campo oportuna, ni toma de muestras para adelantar los análisis en el laboratorio con el fin de determinar los agentes etiológicos acerca de los alimentos implicados con respecto al porcentaje de personas afectadas y alimentos consumidos se detectó que el 30.8\% consumieron alimentos preparados de menor riesgo en salud pública y entre alimentos de mayor riesgo en salud pública como: pollo $16.25 \%$ y $15 \%$ huevos; en cuanto a lugares de consumo el sector turístico seguido de la vivienda ocupan el $37.5 \%$ y el $53.1 \%$ respectivamente. Se concluye que la investigación de los factores determinantes y agentes causales no se ejecutaron adecuadamente.
\end{abstract}

Palabras clave: Enfermedad transmitida por alimentos; Brotes; alimentos de alto riesgo en salud pública, agente etiológico.

Cómo citar:

Montenegro Benavides, M. \& Osorio, P. (2019) COMPORTAMIENTO DE LAS ENFERMEDADES TRANSMITIDAS POR ALIMENTOS Y ESTRATEGIAS DE MEJORAMIENTO EN CUATRO MUNICIPIOS DEL QUINDIO, COLOMBIA. Revista de Investigaciones Universidad del Quindío. Vol 31, pp.62-72. 


\begin{abstract}
During two years, it has been collected data on the epidemiological condition of foodborne diseases in four municipalities of Quindío. During this time, 32 collective cases were presented involving 240 people of different age groups from the cities of Calarcá, Tebaida, Montenegro and Quimbaya. It allowed us to propose strategies to promote the investigation of outbreaks such as the implementation of an epidemiological surveillance strategy. The variables studied were defined according to the notification form of collective cases: gender, age, township, population group, date of exposure, hospitalization, food consumed, signs, symptoms, and laboratory results. In this study, the information recorded in the notification form was analyzed. As a result, we found that the records are incomplete. It demonstrates failures in the continuous information processes of ETA outbreaks. It was not possible to carry out timely field research. As well to take samples to advance the analyzes in the laboratory in order to determine the etiological agents about the food involved in relation to the percentage of people affected and food consumed. However, it was detected that $30.8 \%$ consumed prepared foods of lower risk in public health. Among foods of greater risk in public health the findings are: chicken $16.25 \%$ and $15 \%$ eggs. In regards of consumption locations; $37.5 \%$ at the tourist sector followed by housing $53.1 \%$. It is concluded that the research of determining factors and causative agents were not properly executed.
\end{abstract}

Keywords: Foodborne illness. Outbreaks. High risk food in public health. Etiologic agent.

\section{INTRODUCCIÓN}

A través de los reportes emitidos por el SIVIGILA (Sistema de Vigilancia en Salud Pública) del INSTITUTO NACIONAL DE SALUD DE 2018, el número de brotes de Enfermedades Transmitidas por alimentos con investigación epidemiológica de campo/ por el número total de brotes notificados (población cerrada o cautivaRS) corresponde al $65.5 \%$. ${ }^{[1]}$

Las razones principales por las cuales se necesita investigar los brotes de ETAS son:

- Pueden provocar y afectar la mortalidad de lactantes y niños.

- En los países industrializados afecta al 30\% de la población. ${ }^{[2]}$

- Las consecuencias de las ETA plantean dificultades a los sistemas de salud y reducen la productividad económica.

- Siempre deberá realizarse el análisis socio económico y sanitario de la situación y sus implicaciones nacionales e internacionales. ${ }^{[3]}$

- Deben existir o establecer los mecanismos y los nuevos procedimientos para realizar una investigación. ${ }^{[4]}$

- Se investiga para controlar la propagación y para prevenir futuros brotes.

- Las enfermedades diarreicas transmitidas por alimentos y el agua causan 1.800 .000 muertes por año, especialmente lactantes y niños de países en desarrollo. ${ }^{[5]}$

- La carga de enfermedad en adultos es muy elevada. ${ }^{6]}$

- Actualmente poco se estudia y los sistemas de vigilancia son muy débiles por el incremento de la población mayor. ${ }^{[7]}$

- Incremento de la población inmunodeprimida.

Por la globalización.

- Desplazamiento forzado.

- El incremento e importancia del turismo.

- Las amenazas del Bio-terrorismo.

- Por los cambios de hábitos alimentarios.

- Incremento y manipulación en la venta ambulatoria de alimentos.

Los pasos para la investigación de un brote son: ${ }^{[8]}$

- Determinación de la existencia del brote

- Confirmación del diagnóstico

- Determinación del número de casos

- Organización de la información en términos de tiempo, lugar y persona.

- Determinación de las personas que están en riesgo de enfermarse.

- Formulación de Hipótesis.

- Análisis de los datos.

- Establecimiento de las medidas de control.

- Elaboración del Análisis y establecimiento de 
recomendaciones.

- Informe final del nivel local a los otros niveles. [9]

En el Departamento del Quindío no se ejecutan la totalidad de los pasos para la investigación de un brote.

Es así como se hacen necesarias las acciones de inspección, vigilancia y control de alimentos, orientar medidas de prevención y control de los alimentos en los diferentes municipios del Departamento, colaborando con la organización y el análisis de la información a través de un seguimiento continuo y sistemático de las enfermedades transmitidas por alimentos de acuerdo con los procesos establecidos para la notificación, recolección y análisis de los datos, que permitan detectar los alimentos, agentes etiológicos y factores contribuyentes a su transmisión y así generar información oportuna, válida y confiable. ${ }^{[10,11]}$

Para realizar el diagnóstico actual del Departamento se hizo necesario entonces realizar un estudio descriptivo comparativo retrospectivo, del total de eventos colectivos completos registrados en los municipios de Montenegro, Quimbaya, Calarcá y La Tebaida, durante dos años, tomando como unidad muestral la Ficha de notificación de casos colectivos para Enfermedades Transmitidas por Alimentos y estudiando las propiedades o características de las personas afectadas como son: género, edad, municipio, procedencia, ocupación, régimen de salud, grupo poblacional, pertenencia étnica, fecha de inicio de síntomas, fecha de exposición, clasificación inicial del caso, hospitalización, intoxicación, síntomas y resultados de laboratorio. ${ }^{[12]}$

Las Enfermedades Transmitidas por Alimentos se producen cuando se ingieren en los alimentos cantidades suficientes de agentes químicos o microorganismos patógenos y/o sus toxinas. Frecuentemente se asocian a la ingesta de un alimento deficientemente preparado o mal conservado. ${ }^{[13]}$ La Enfermedad transmitida por alimentos, es el síndrome originado por la ingestión de alimen- tos o agua, que contengan agentes etiológicos en cantidades tales que afecten la salud del consumidor a nivel individual o a grupos de población. Las alergias por hipersensibilidad individual a ciertos alimentos no se consideran ETA. ${ }^{[15]}$

Se han descrito más de 250 enfermedades diferentes transmitidas por los alimentos. La mayoría de estas enfermedades son infecciones, ocasionadas por distintas bacterias, virus y parásitos que pueden ser transmitidos por los alimentos. Otras enfermedades son envenenamientos, ocasionados por toxinas o productos químicos nocivos que han contaminado los alimentos, por ejemplo, hongos venenosos. Estas diferentes enfermedades tienen muchos síntomas diferentes. El microbio o toxina se introduce en el cuerpo a través del conducto gastrointestinal, y a menudo ocasiona los síntomas tales como náuseas, vómito, calambres abdominales y diarrea, síntomas comunes en muchas enfermedades transmitidas por los alimentos. ${ }^{[16,17,18]}$

Los brotes de enfermedades de origen alimentario por lo común se caracterizan por la aparición del cuadro clásico en un lapso breve, aunque variable (de horas o semanas), después del consumo, entre personas que han comido los mismos alimentos. Es esencial el estudio de laboratorio rápido y detenido de todos los casos y de los alimentos implicados. Es difícil identificar los casos aislados de enfermedad de origen alimentario. La enfermedad de origen alimentario puede ser una de las causas más comunes de un cuadro clínico agudo, sin embargo, muchos casos y brotes pasan inadvertidos y no se notifican. ${ }^{[19,20]}$

Cada año 77 millones de personas enferman y más de 9.000 mueren en las Américas a causa de enfermedades de transmisión alimentaria. Las enfermedades diarreicas representan el $95 \%$ de las enfermedades de transmisión alimentaria en la región. ${ }^{[21]}$ La primera estimación de la carga mundial de las enfermedades de transmisión alimentaria muestra que casi 1 de cada 10 personas enferman cada año al ingerir alimentos contaminados y 420.000 mueren como consecuencia de 
estas enfermedades. ${ }^{[22]}$ Los niños corren un riesgo especial de padecer enfermedades diarreicas transmitidas por los alimentos: 220 millones enferman y 125.000 mueren cada año. ${ }^{[23]}$

Las enfermedades diarreicas causan más de la mitad de la carga mundial de las enfermedades de transmisión alimentaria, con 550 millones de personas que enferman y 230.000 que mueren cada año. La diarrea suele deberse a la ingestión de carne y huevos crudos o mal cocidos, verduras y frutas mal lavadas, y productos lácteos, contaminados por Norovirus, Campylobacter, Salmonella no tifoídica y Escherichia coli patógena. ${ }^{[24,25]}$

El riesgo de padecer enfermedades de transmisión alimentaria es mayor en los países de ingresos bajos y medianos, y está vinculado a la preparación de alimentos con agua contaminada, la falta de higiene y condiciones inadecuadas en la producción y el almacenamiento de alimentos, el bajo nivel de alfabetismo y educación, y la insuficiencia de leyes en materia de inocuidad de los alimentos o su falta de aplicación. ${ }^{[26,27]}$

Las enfermedades de transmisión alimentaria pueden causar síntomas de corta duración, como náuseas, vómitos y diarrea (afección que generalmente se denomina "intoxicación alimentaria"), pero también pueden causar enfermedades más prolongadas, como cáncer, insuficiencia renal o hepática y trastornos cerebrales y neurales. Estas enfermedades pueden ser más graves en los niños, las embarazadas, los ancianos y las personas con el sistema inmunitario debilitado. Los niños que sobreviven algunas de las enfermedades de transmisión alimentaria más graves pueden sufrir retraso del desarrollo físico y mental, que tiene efectos irreversibles en su calidad de vida. ${ }^{[28,29]}$

Por lo anterior, es necesario describir la condición epidemiológica de los brotes de Enfermedades Transmitidas por alimentos en cuatro municipios del Departamento del Quindío, para proponer estrategias que permitan la mejora en la investigación de los brotes. ${ }^{[30]}$

\section{MATERIALES Y MÉTODOS}

Esta investigación permitió evaluar todos los eventos colectivos completos de los municipios de Montenegro, Quimbaya, Calarcá, y Tebaida del Departamento del Quindío, Colombia durante dos años.

Estos eventos fueron estudiados mediante un análisis univariado y bivariado de los datos contenidos en el reporte realizado por las Instituciones Prestadoras de Salud de cada Municipio por medio de las fichas de notificación de casos colectivos para Enfermedades Transmitidas por Alimentos, elaborada por el Instituto Nacional de Salud y el Ministerio de Salud, Sistema Nacional de Vigilancia en Salud Pública, Subsistema de información; que a la vez son enviadas a la secretaria de Salud del Quindío.

La investigación tomó en cuenta solo los casos colectivos completos, o sea los eventos que contenían ficha colectiva, ficha individual de los implicados, resultados de muestras de alimentos, actas de visita realizada por los funcionarios de la Secretaría de Salud del Quindío. Se excluyeron de la investigación los casos no notificados a la Secretaría de Salud del Quindío, los casos individuales y casos de otros Municipios.

Se determinaron las variables de los individuos implicados en los eventos: género, edad, municipio, procedencia, ocupación, régimen de salud, grupo poblacional, pertenencia étnica, fecha de inicio de síntomas, fecha de exposición, clasificación inicial del caso, hospitalización, intoxicación, síntomas, resultados de laboratorio y de alimentos.

Una vez recolectada esta información, se creó una base de datos en Microsoft Office Excel plus 2016 la cual podrá seguir siendo alimentada en la Secretaría de Salud del Quindío. Se analizó la información realizando tablas comparativas, y gráficas que permitieron llegar a las conclusiones y recomendaciones finales. 


\section{RESULTADOS}

En total se reportaron 32 eventos de ETAS, 17 casos durante el primer año y 15 durante el segundo año, ocurridos en los municipios de $\mathrm{La}$ Tebaida, Montenegro, Quimbaya y Calarcá, los cuales después de la capital, son los más turísticos y poblados del Quindío, como se presenta en la gráfica 1 .

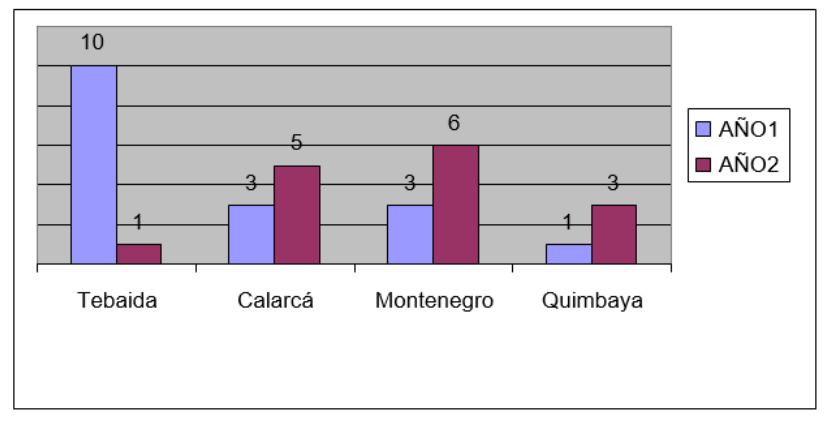

Gráfica 1. Distribución de casos colectivos según municipio y año.

La distribución por año arrojó que en el primer año el mayor número de eventos colectivos se presentó en el municipio de La Tebaida, los cuales representaron el 58.8\% del total; para el año 2, fue el municipio de Montenegro quien tuvo mayor número, con el $40 \%$.

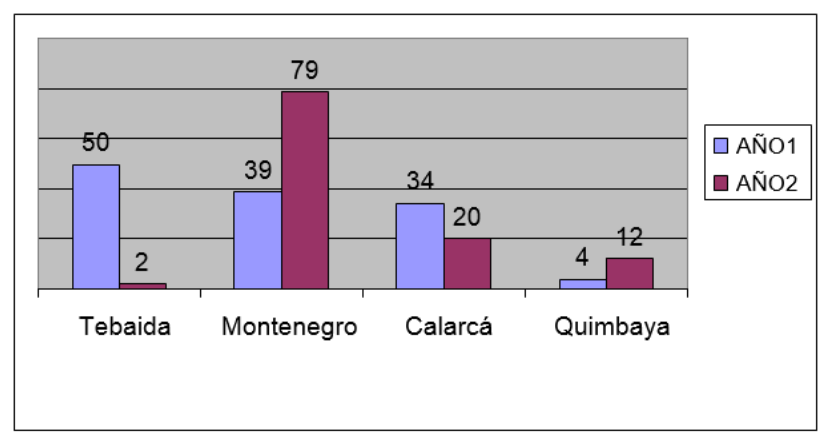

Gráfica 2: Distribución de casos según número de personas implicadas, municipio y año.

El total de personas comprometidas por Enfermedades Transmitidas por alimentos fueron 240, distribuidas así: 127 personas en el año uno y 113 personas en el año dos, las cuales representaron el $52.9 \%$ y el $47.1 \%$ respectivamente. (gráfica 2).

Durante el año uno el municipio con más personas implicadas fue La Tebaida; allí se afectaron
50 personas, lo cual representó el $39.3 \%$ con respecto al total de casos. Para el año dos fue el municipio de Montenegro donde se presentaron 79 casos lo cual corresponde al $69.9 \%$ con respecto al total.

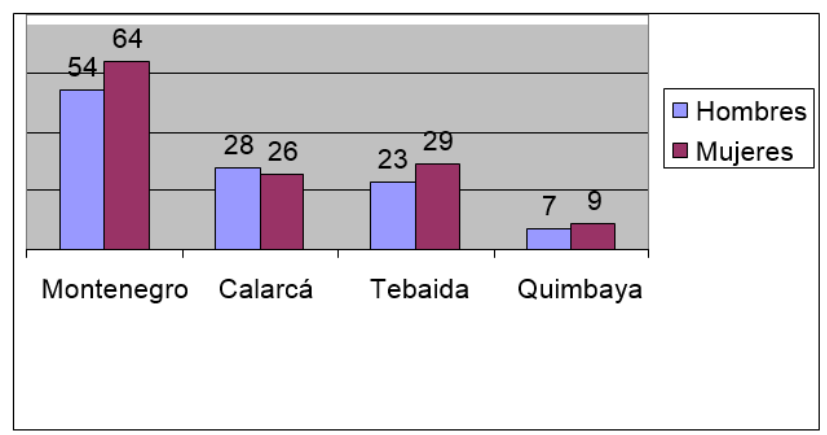

Gráfico 3. Distribución de casos personas implicadas según género y municipio.

De las 240 personas involucradas en ETAS el $53.3 \%$ (128) pertenecían al género femenino y el $47 \%$ (112) al género masculino. Podemos decir que ésta distribución fue prácticamente igual en los municipios estudiados a diferencia del municipio de Calarcá donde se reportaron mayor número de casos para el género masculino. (gráfico $3)$.

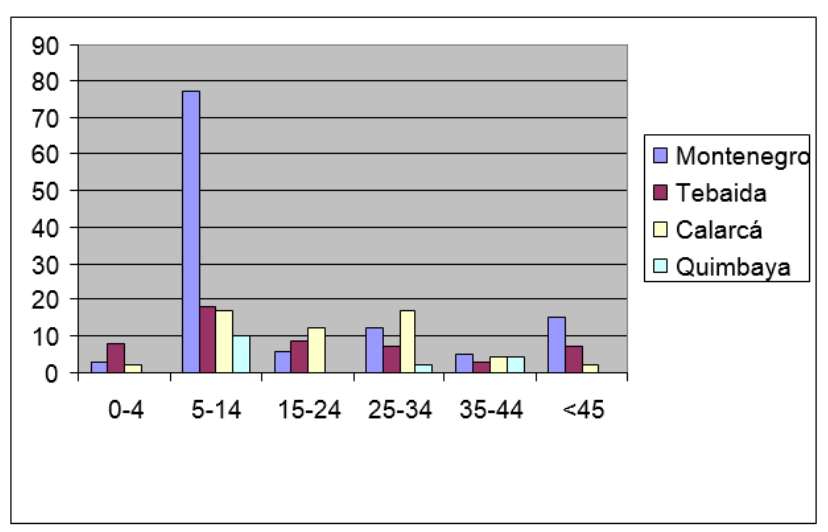

Gráfico 4: Distribución de casos según edades y municipio.

En los cuatro municipios estudiados, se pudo observar que el grupo de edad más afectado fue el de personas entre los 5 y 14 años (gráfico 4); de acuerdo al reporte, corresponde a viajeros turistas que visitaron los lugares del Quindío. 


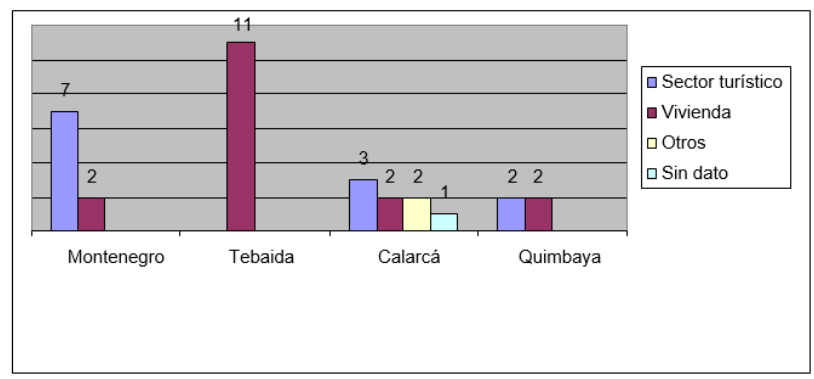

Gráfica 5: Distribución de casos colectivos según municipios y lugares de consumo.

En el municipio de Montenegro se observó que el sector turístico seguido de la vivienda ocupan los lugares de consumo con más casos colectivos, afectando a 112 personas en el sector turístico representando el $37.5 \%$ con respecto al total de casos y a 6 personas en las viviendas representando el 53.1\% del total; el municipio de Montenegro al igual que Calarcá cuenta con sitios como parques temáticos y fincas agro turísticas que se frecuentan constantemente, lo que aumenta el riesgo para que ocurra un evento de ETA.

Los sitios de vivienda que involucraron a 52 personas que se presentaron en La Tebaida corresponden a barrios donde fueron reubicados las personas afectadas por el terremoto de 1999 por lo tanto son lugares con escasez de recursos económicos y socioculturales; en donde persisten los problemas de saneamiento básico como: agua potable y servicios públicos, razón por la cual no se aplican hábitos higiénicos saludables convirtiéndose en un factor determinante para presentarse un brote de ETA.

Se observó que, en el Municipio de Calarcá, los lugares de consumo donde se presentan más casos colectivos fueron los lugares turísticos: estaderos, restaurantes, hoteles, ocupando el 37.5\% ya que el municipio cuenta con eventos a nivel nacional como el reinado nacional del café y lugares turísticos, además, es pionero en el servicio de alojamiento rural. Todo esto hace que sean los lugares de consumo más implicados.

Para Calarcá los lugares de consumo se registraron con la variable otros, que corresponde a Co- legio y penitenciaria. Involucrando 25 personas. En sector turístico se involucraron 15 personas. Sin dato, se refiere, a que no se registró el lugar de consumo en la ficha de notificación, involucrando 9 personas, en el dato de vivienda se registraron 5 personas.

De acuerdo con los lugares de consumo del municipio de Quimbaya se observó que los casos colectivos son iguales en vivienda y en sitio turístico, pero en cuanto a las personas involucradas se reportó 12 en sector turístico y 4 personas en vivienda; el municipio de Quimbaya cuenta con parque temático y lugares turísticos encontrando que estos sitios fueron los más involucrados en los casos de ETAS. Comparando los municipios Montenegro y Quimbaya se observó que, a pesar de que los municipios son similares en población y en cercanía a los sitios turísticos, Montenegro reportó más casos de ETAS que Quimbaya, debido posiblemente a que muchas personas se hospedan en Montenegro, pero consumen en Quimbaya, por lo tanto, se notifican de Montenegro como lugar de origen.

En Calarcá se percibió que los lugares de consumo se encontraron el sector turístico como: hoteles, fincas agro-turísticas, parques temáticos, entre otros, los cuales pueden presentar varios factores de riesgo como: Contaminación cruzada en el sitio donde se preparan los alimentos, incumplimiento de BPM (Buenas Prácticas de Manufactura) en el momento de preparar los alimentos y en el establecimiento, no se aplica temperaturas de refrigeración constante para los lácteos en el sitio del evento, entre otras.

En el municipio de Montenegro se encuentran distribuidas a nivel rural gran parte de alojamientos y fincas agro-turísticas que solicitan el servicio a domicilio en los supermercados del municipio quienes se encargan de transportar los alimentos perecederos como pollo y lácteos a temperatura ambiente, interrumpiendo la cadena de frío que deben mantener dichos alimentos para evitar el crecimiento microbiano, convirtiéndose en un factor de riesgo para ETAS. 
En el municipio de La Tebaida se encontró que los lugares de consumo son viviendas las cuales tienen problemas de saneamiento básico que se relacionan con la elaboración de los alimentos sobre todo en festividades de fin de año, en donde no se aplican buenas condiciones higiénicas para la preparación de esos alimentos. (gráfica 5).

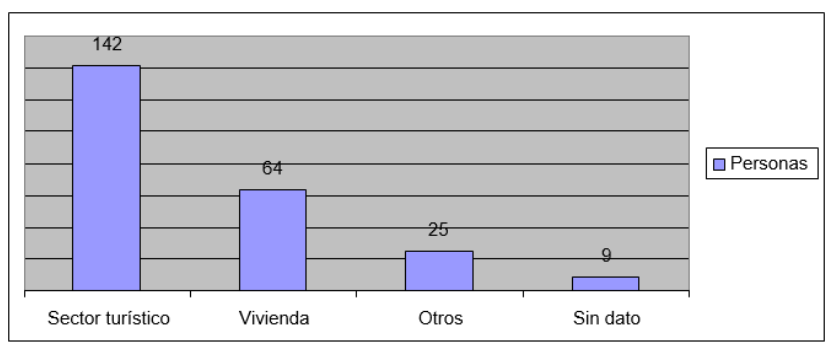

Gráfica 6: Distribución de casos según lugar de consumo y personas afectadas.

De las 240 personas involucradas en eventos de ETAS (gráfica 6), 142 (59.1\%) corresponden a lugares de consumo del sector turístico. Una vez analizado el rango de edades jóvenes entre 5 y 14 años, se concluye que son personas incluidas en los planes turísticos ofrecidos a grupos de colegios y grupos organizados.

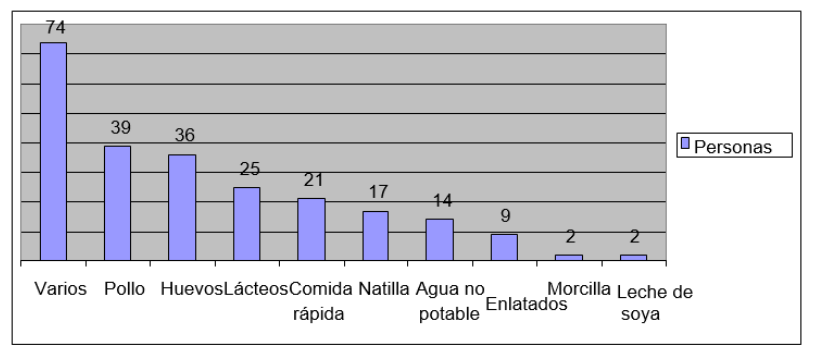

Gráfica 7: Distribución de personas afectadas según alimento.

Con respecto al porcentaje de personas afectadas y alimentos consumidos se encontró que: El mayor número de personas 74 (30.8\%) consumieron alimentos designados como varios en los que se incluyen: desayunos, almuerzos, cenas, y que están compuestos por diversos elementos como: chocolate, arepa, leguminosas: frijoles, lentejas; carnes, arroz con pollo, sopas, jugos, ensaladas, comidas típicas del Departamento del Quindío y que se ofrecen en los sitios turísticos de los municipios implicados.
Le sigue el pollo que lo consumieron 39 personas (16.25\%) y los huevos por 36 personas (15\%) los cuales se consumieron en las viviendas, en donde posiblemente no se tuvo la precaución de realizar un lavado y desinfección de estos alimentos que están involucrados en contaminación por salmonella ocasionando la salmonelosis clasificada como Enfermedad Transmitida por alimentos.

Otros alimentos que aparecieron comprometidos son los lácteos los cuales corresponden a la clasificación de alimentos perecederos, que deben conservarse a temperatura de refrigeración, además, en los municipios del Quindío se presenta la problemática del consumo de leche cruda y elaboración de derivados lácteos con leche cruda, lo cual hace que su carga microbiana se dispare y se convierta en un riesgo para ETAS.

Al analizar los alimentos implicados, de acuerdo al municipio, se encontró que para el municipio de Calarcá se encuentran en el sector de varios y lácteos que corresponden a una mezcla de desayunos, almuerzos o comidas consumidas en los sitios turísticos, con un 37.5\% para alimentos preparados y $25 \%$ para lácteos y pollo lo que demuestra que los alimentos perecederos son los alimentos que presentan mayor factor de riesgo en el caso de una ETA.

El total de los casos colectivos en Montenegro fueron 9 los cuales se distribuyeron de acuerdo con los alimentos consumidos así: 44.4\% pollo, $22.2 \%$ lácteos y $11.1 \%$ para agua no potable, comidas rápidas y varios, demostrando que los alimentos de alto riesgo en salud pública como pollo y lácteos ocupan la mayor distribución de los casos colectivos.

El $54.5 \%$ de los alimentos consumidos en los casos colectivos correspondieron a lácteos que se consumieron en las viviendas de La Tebaida como: leche pare elaborar chocolate, quesos, los cuales utilizan en su gran mayoría leche cruda sin pasterizar aumentando el riesgo de contener microorganismos patógenos como coliformes fecales, Staphilococcus Coagulasa positiva, además, 
en este municipio se puede incrementar el riesgo de contaminación de alimentos perecederos porque su promedio de temperatura corresponde al más elevado del Departamento.

En el municipio de Quimbaya se observó que los alimentos comprometidos son comidas rápidas como: hamburguesa, perro caliente, carnes frías, los cuales se elaboran en su gran mayoría en ventas ambulantes, los cuales se catalogan como establecimientos de alto riesgo epidemiológico ya que sus condiciones higiénico-sanitarias no cumplen con lo establecido en la normatividad vigente; por lo tanto, amerita realizar acciones de Inspección, Vigilancia y control.

En el municipio de Quimbaya se puede observar que el sector turístico ocupa una mayor distribución de los casos colectivos, ya que la mayoría de turistas van y consumen en los parques temáticos y si presentan síntomas por su cercanía consultan en este municipio, el cual realiza su respectivo reporte de ETA. (gráfica 7).

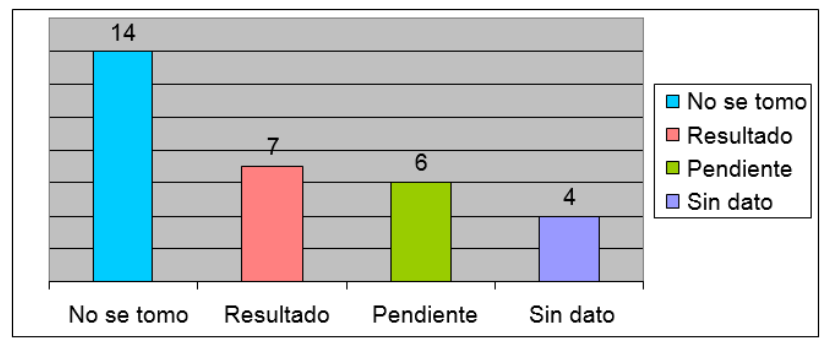

Gráfico 8: Distribución de casos colectivos según muestras de laboratorio reportadas.

En cuanto a la confirmación del agente etiológico por parte del laboratorio al $43.8 \%$ de los casos (14), no se tomaron muestras de laboratorio para su confirmación (gráfica 8). Al laboratorio fueron remitidos para su confirmación 28 casos $(56.2 \%)$, de los cuales se reportó resultado para 7 de ellos, cuyo diagnóstico fue negativo para agentes contaminantes, para 6 casos el resultado aparece pendiente, pero no fue reportado a la ficha de notificación ni al reporte consolidado de la investigación epidemiológica y para los 4 casos restantes no aparece ningún dato en la ficha de notificación, lo que demuestra fallas en los procesos de información continua y oportuna de casos y brotes de ETA, no se hizo posible que se realice investigación de campo oportuna, ni se establece si se trata de casos aislados o de brotes, no se realizó la toma de muestras para adelantar los análisis en el laboratorio con el fin de determinar el agente causante, e intervenir oportunamente, ya que al no tomar muestras de alimentos por parte de los Técnicos de saneamiento o muestras biológicas a las personas comprometidas por parte de las EPS (Entidades Prestadoras de Salud) no permite identificar el agente etiológico causante de la ETA y la investigación queda inconclusa.

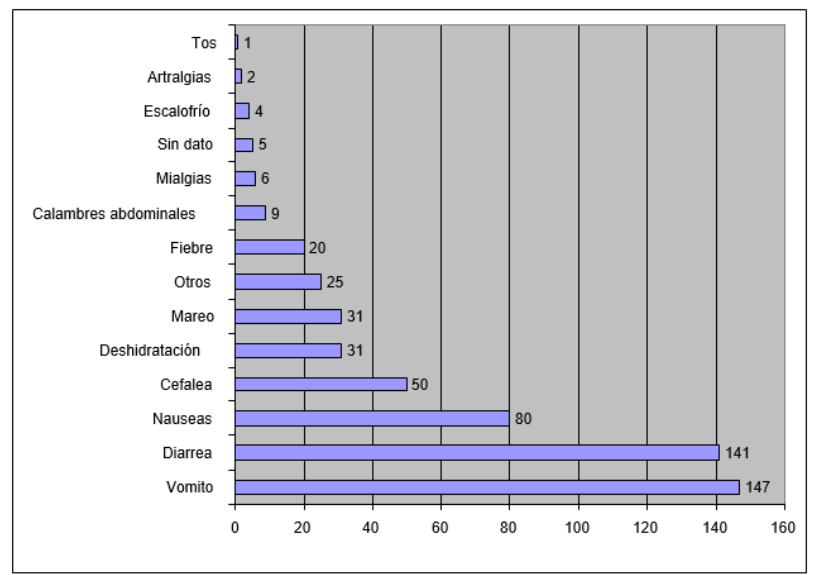

Gráfica 9: Distribución según sintomatología.

Teniendo en cuenta los signos y síntomas presentados en los casos de ETAS durante los años $1 \mathrm{y}$ 2 (gráfica 9), se observó que los más representativos son: vómito, diarrea, nauseas y cefalea los cuales pudieron corresponder a una fuente o agente etiológico como: agentes fúngicos, agentes químicos: intoxicación por cadmio, intoxicación por estaño, intoxicación por zinc, intoxicación por órgano fosforados, intoxicación por hidrocarburos clorados, Agentes bacterianos: Bacillus cereus, intoxicación estafilocócica, diarrea por aeromonas, campylobacteriosis, cólera, gastroenteritis por Escherichia coli patógena, salmonelosis, listeriosis ; agentes víricos: gastroenteritis vírica.

Los agentes etiológicos no se pudieron caracterizar en los brotes de ETAS analizados en los años 1 y 2 respectivamente, debido a que se necesitaba que en la ficha de notificación se especificará el 
periodo de incubación de dicho agente, se necesitaba analizar los resultados de los análisis de muestras de alimentos, biológicas, o muestras de superficie para determinar el tipo de microorganismo que pudo causar el brote de ETA.

A través de los informes elaborados por el grupo de funcionarios encargados de la vigilancia e investigación de los eventos y enviados por algunos municipios como Calarcá y La Tebaida, se pudo observar que los factores de riesgo más frecuentes son: Contaminación cruzada, higiene personal, manipulador infectado, pérdida de cadena de frío, en general debilidades en Buenas Prácticas de Manufactura contempladas en la Resolución 2674 de 2013.

\section{DISCUSIÓN}

La investigación se fundamentó en los reportes presentados por los funcionarios de la Secretaría de Salud del Quindío y en las fichas de notificación obligatoria de casos colectivos para enfermedades transmitidas por alimentos de cada una de las IPS ( instituto prestador de servicios de salud) de los municipios estudiados encontrando que los factores de riesgo de estos eventos se han dado por contaminación cruzada, mala higiene personal, manipulador infectado, pérdida de cadena de frío de esta misma manera se identificó que las personas afectadas son las mujeres cuyas edades estuvieron contempladas entre 5-14 años. ${ }^{[1]}$

Con relación a los alimentos implicados en el estudio, las personas afectadas de brote de ETA en los cuatro municipios durante dos años son: varios alimentos preparados en vivienda (chocolate, arroz, jugo, ensalada, carnes, entre otros) y pollo, considerados en la Resolución 2674 de 2013, como alimentos de mayor riesgo en salud pública; pero esto va relacionado de acuerdo al análisis realizado a lugares de consumo como establecimientos turísticos y la vivienda, identificando estos como los lugares de mayor riesgo para que ocurra una ETA. ${ }^{[3]}$
Respecto al agente etiológico, no se identificaron microorganismos patógenos en muestras de alimentos y biológicas debido a varios factores como: Fallas en el proceso de investigación y en el análisis oportuno de las muestras tomadas, en muchos casos no se tomaron muestras de alimentos ni biológicas en el evento. ${ }^{[4]}$

Durante dos años, se presentaron más eventos colectivos en los municipios de La Tebaida y Montenegro; pero no podemos hacer a un lado los reportes significativos para cada municipio investigado, llegando a la conclusión de que en el Departamento del Quindío no se cumple con lo enunciado en el protocolo de ETA, haciéndose necesario fortalecer la investigación de un brote de ETA a través del personal de salud y que se apliquen completamente los pasos del protocolo para que los casos investigados se puedan concluir satisfactoriamente. ${ }^{[9]}$

Las Instituciones Prestadoras de Salud deben registrar oportunamente y en su totalidad los datos en los formatos suministrados por las entidades territoriales, con el fin de evitar subregistro o datos incompletos que perjudican la investigación. [10]

\section{AGRADECIMIENTOS:}

-Secretaría de Salud del Quindío

- Patricia Zapata qepd.

Docente Fundación Universitaria del Área Andina Seccional Pereira.

-Carmen Luisa Betancur

Docente Dirección de posgrados. Investigadora Asociada Colciencias 2019, líder grupo ZIPATEFI 


\section{REFERENCIAS BIBLIOGRÁFICAS}

1. FAO/OMS. PROGRAMA CONJUNTO FAO/OMS SOBRE NORMAS Instituto Nacional de Salud. Protocolo de Vigilancia de Enfermedades Transmitidas por Alimentos. SIVIGILA Colombia. 2018. publicado en: http://portalsivigila.ins.gov.co/sivigila/documentos/Docs_1.php.

2. Rodríguez Torrens, Herlinda; Barreto Argilagos, G.; Sedrés Cabrera, Martha; Bertot Valdés, J.; Martínez Sáez, S.; Guevara Viera, G. Las enfermedades transmitidas por alimentos, un problema sanitario que hereda e incrementa el nuevo milenio REDVET. Revista Electrónica de Veterinaria, vol. 16, núm. 8, 2015, pp. 1-27 Veterinaria Organización Málaga, España.

3. FAO/OMS. PROGRAMA CONJUNTO FAO/OMS. Codex Alimentarius: proteger la salud, facilitar el comercio. Organización de las naciones unidas para la alimentación y la agricultura. 2019. Publicado en: http://www.fao.org/fao-stories/article/es/c/1096383/

4. Organización Panamericana de la Salud. Instituto panamericano de protección de alimentos y zoonosis. Guía VETA Guía para el establecimiento de sistemas de vigilancia epidemiológica de Enfermedades Transmitidas por alimentos (VETA) y la investigación de brotes de Toxi-infecciones alimentarias. Ministerio de Salud. Bogotá. Publicado en: www.paho.org/hq/index.php?option=com content\&view= article\&id=10540:2015-capitulo-i-guia-veta-inocuidad-de-alimentos\&Itemid=41414\&lang=es. Consulta julio 2019.

5. Organización Panamericana de la Salud. Vigilancia y prevención de ETAS. [sitio en internet]. Disponible en: http:/ed.OPS/HCP/HCV/FOS/103/96 Consulta 20 de agosto de 2008.

6. Gutiérrez Miriam. Toxicología alimentaria y principales tóxicos en alimentos. En: Seminario-Taller Internacional de Enfermedades Transmitidas por Alimentos; Bogotá. 15-17 de octubre de 2008.Bogota: Instituto Nacional de Salud, 2008.

7. Centros para el control y la prevención de enfermedades. CDC Departamento de Salud y servicios humanos. Enfermedades transmitidas por alimentos. [sitio en internet]. Disponible en: Htpp://www. foodsafety.gov. consulta septiembre 2008.

8. Gobernación del Quindío. Municipios del Quindío. [sitio en internet]. Disponible en: http://www. quindio.gov.co/home/index.php. consulta: junio 2019.

9. Veta y la Investigación de Brotes de Toxi-Infecciones Alimentarias. Programa de Salud Pública Veterinaria. [sitio en internet]. Disponible en: https://www.assal.gov.ar/assa/userfiles/file/guia\%20veta. pdfconsulta: junio 2019.

10. Red de vigilancia epidemiológica de Castilla y León. Programa de vigilancia para el control de los brotes de Enfermedades transmitidas por agua y alimentos año 2013.

11. W. C. Frazier y D. C. Westhoff. Microbiología de los alimentos. 4 ed. España: Editorial Acribia; 1993.

12. Epidemiology of Foodborne Illness. Saulat Jahan Research and Information Unit Primary Health Care Administration, Qassim Ministry of Health Kingdom of Saudi Arabia. [sitio en internet]. Disponible en: http://cdn.intechopen.com/pdfswm/27392.pdf. Consulta: mayo 2019.

13. Andrea O, Janepsy D, Rodrigo F, Alejandra V, Maritza G. Vigilancia de brotes de enfermedades transmitidas por alimentos en Chile. Rev. Chilena Infectol 2012; 29 (5): 504-510.

14. World Health Organization Global Estimates and Regional Comparisons of the Burden of Foodborne Disease in 2010. Havelaar AH, Kirk MD, Torgerson PR, Gibb HJ, Hald T, et al. (2015) World Health Organization Global Estimates and Regional Comparisons of the Burden of Foodborne Disease in 2010. PLoS Med 12(12): e1001923. doi: 10.1371/journal.pmed.1001923.

15. Organización Mundial de la Salud. Enfermedades de transmisión alimentaria, temas de salud. [sitio en internet]. Disponible en: http://www.who.int/topics/foodborne_diseases/es/.consulta: mayo 2019.

16. Organización Mundial de la Salud. Estimaciones de la OMS sobre la carga mundial de enfermedades de transmisión alimentaria. [sitio en internet] Disponible en: www.who.int/foodsafety/publications/ foodborne disease/fergreport/es/. 2015 Consulta junio de 2019.

17. Organización Mundial de la Salud. Guía para los viajeros sobre la inocuidad de los alimentos [sitio en internet] Disponible en: www.who.int/foodsafety/publications/foodborne_disease/fergreport/es/Consulta junio de 2019.

18. Organización Mundial de la Salud. Datos y cifras sobre las enfermedades de transmisión alimentaria. [sitio en internet] Disponible en: www.who.int/foodsafety/areas_work/foodborne-diseases/ferg_infographics/es/ consulta junio 2019.

19. Organización Mundial de la Salud. Manual sobre las cinco claves para la inocuidad de los alimentos. [sitio en internet] Disponible en: www.who.int/foodsafety/publications/foodborne_disease/fergreport/ 
es/ Consulta junio de 2019.

20. Organización Mundial de la Salud. Inocuidad de los alimentos, temas de salud. [sitio en internet]. Disponible en: http://www.who.int/topics/food_safety/es/ Consulta: mayo 2019.

21. Espinosa L., Varela C., Martínez, E V., Cano, R. Brotes de enfermedades transmitidas por alimentos (excluye brotes hídricos). Boletín epidemiológico semanal. 2014. [ sitio en internet] Disponible en: http://revista.isciii.es/public/journals/1/pdf 195.pdf Consulta: junio 2019.

22. Red Nacional de Vigilancia Epidemiológica Instituto de Salud Carlos III. Resultados de la Vigilancia Epidemiológica de las Enfermedades Transmisibles; Informe Anual, Año 2012. [sitio en internet]. Disponible en: http://gesdoc.isciii.es/gesdoccontroller?action=download\&id=21/01/2015-3962d0c4cd. Consulta: mayo 2019.

23. Organización Mundial de la Salud. Informe de la OMS señala que los niños menores de 5 años representan casi un tercio de las muertes por enfermedades de transmisión alimentaria. 2015. [sitio en internet] Disponible en: www.who.int/foodsafety/areas_work/foodborne diseases/ferg_infographics/ es/ consulta junio 2019.

24. A cross-sectional study on the microbiological quality and safety of raw chicken meats sold in Nairobi, Kenya. BMC Res Notes. 2014; 7(1): 627.

25. Informe Técnico Sobre Ingeniería Agrícola Y Alimentaria. Enfermedades transmitidas por alimentos y su impacto socioeconómico. Estudios de caso en Costa Rica, El Salvador, Guatemala, Honduras y Nicaragua. [sitio en internet]. Disponible en: http://www.fao.org/3/a-i0480s.pdf. Consulta: mayo 2019.

26. Plan decenal de salud pública. Ministerio de salud y Protección social, dimensión seguridad alimentaria y nutricional.2010 - 2021. [sitio en internet]. Disponible en: http://www.minsalud.gov.co/plandecenal/Documents/dimensiones/dimensionseguridadalimentariaynutricional.pdf. Consulta: mayo 2019.

27. Puig Yamila, Maceo Robert, Brady Antonia, Leyva Virginia. Factores Epidemiológicos de Interés en Brotes de Enfermedades Transmitidas por Alimentos en la Habana. Revista cubana de Higiene y epidemiologia.2013. [sitio en internet]. Disponible en http://scielo.sld.cu. Consulta: mayo 2019.

28. Estimaciones sobre enfermedades transmitidas por alimentos en los EE. UU. en el 2011. [sitio en internet]. Disponible en: http://www.cdc.gov/spanish/Datos/EnfermedadesAlimentos/. Consulta: junio 2019.

29. CrimStacy, Crim M, Iwamoto M, Huang JY, Griffin PM, Gilliss D. Incidence and trends of infectionwithpathogenstransmittedcommonlythroughfood - FoodborneDiseases Active Surveillance Network, 10 US sites, 2006- 2013.Revista MMWR Morb Mortal WklyRep. 2014. volumen 63(15):328-332. [sitio en Internet] disponible en: http://www.cdc.gov/. Consulta: junio 2019.

30. De la Hoz Gómez, Erika Andrea, Paba Osorio, Baldir. comportamiento epidemiológico de las enfermedades transmitidas por alimentos (eta) en el departamento de sucre durante el periodo $2012-2015$. Santa Marta 2016. [sitio en internet]. Disponible en: http://repository.ucc.edu.co/bitstream/ucc/742/1/ Especializaci\%C3\%B3n\%20en\%20epidemiolog\%C3\%ADa.-.pdf. Consulta: junio 2019. 